\title{
Anomalous Left Coronary Artery Originating from the Right Coronary Sinus with an Interarterial Course: a Case Report and Literature Review
}

\author{
Judit Simon ${ }^{1}$, Alexisz Panajotu ${ }^{1}$, Judit Csőre ${ }^{1}$, Miklós Pólos ${ }^{1}$, Emese Zsarnóczay ${ }^{1}$, Béla \\ Merkely ${ }^{1}$, Pál Maurovich-Horvat ${ }^{1,2}$ \\ ${ }^{1}$ MTA-SE Cardiovascular Imaging Research Group, Heart and Vascular Center, Faculty of Medicine, Semmelweis University, \\ Budapest, Hungary \\ ${ }^{2}$ Medical Imaging Centre, Faculty of Medicine, Semmelweis University, Budapest, Hungary
}

\section{ABSTRACT}

Introduction: The diagnosis of coronary artery anomalies (CAAs) can be challenging due to the highly variable manifestations and symptoms. CAAs occur in less than $1 \%$ of the population. Multidetector cardiac computed tomography angiography (CTA) provides excellent spatial and temporal resolution for the imaging of CAAs. This case report describes an anomalous origin of the left main coronary artery. Case presentation: A 64-year-old woman with atypical chest pain underwent coronary CTA. The CT demonstrated that the left coronary artery was originating from the right coronary sinus and had an interarterial course with an approximately $50 \%$ stenosis at the segment between the great arteries. Invasive coronary angiography confirmed hemodynamically significant stenosis of the interarterial segment. The patient underwent off-pump coronary artery bypass graft surgery. Conclusion: Left coronary artery originating from the right coronary sinus with an interarterial course increases the risk of sudden cardiac death. In these patients, surgical revascularization is recommended. However, timely diagnosis, especially in those with mild symptoms, remains challenging. Coronary CTA is a robust tool to diagnose CAA and provides valuable information to support the clinical decision making in this patient population.

Keywords: coronary artery anomaly, computed tomography angiography

\section{ARTICLE HISTORY}

Received: April 2, 2020

Accepted: May 4, 2020

\section{CORRESPONDENCE}

Judit Simon

Gaál József út 9

1122 Budapest, Hungary

Tel: +3614586756

E-mail: juditsimon21@gmail.com

\section{INTRODUCTION}

Coronary artery anomalies (CAAs) are seen in less than $1 \%$ of the general population. ${ }^{1,2}$ Since manifestations and pathophysiological mechanisms are highly variable, CAAs are a very diverse group of congenital heart disorders. CAAs are traditionally classified based on the origin, course, and termination of the anomalous vessel. ${ }^{3}$ Anomalies of the origin have three subcategories: absent left main (LM) artery, anomalous ostium outside of the aortic sinuses, 


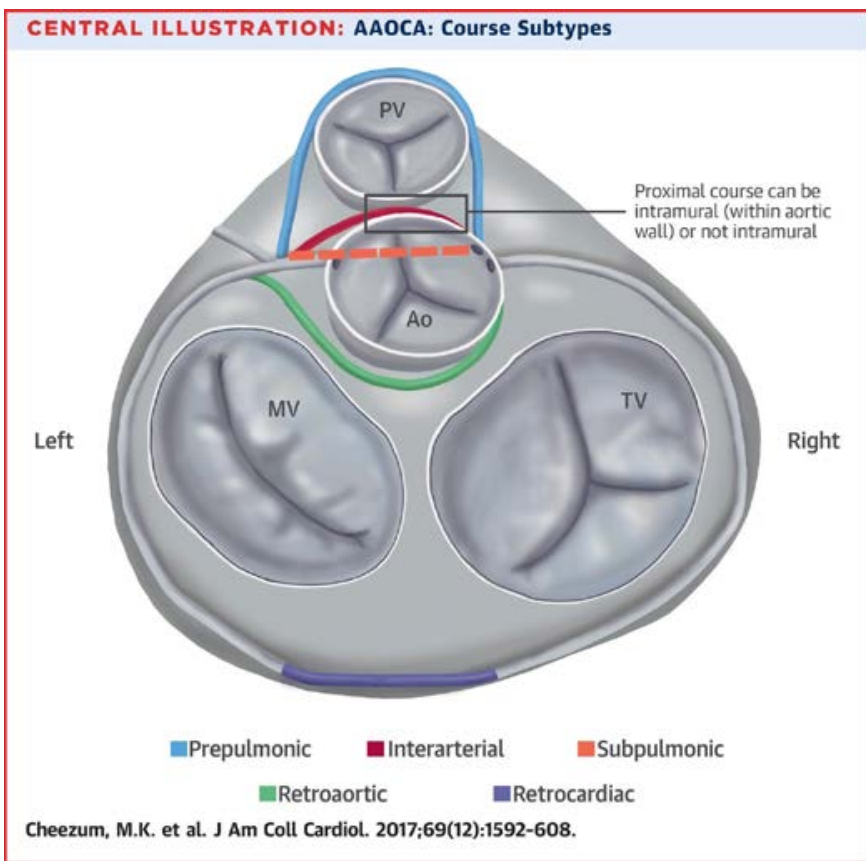

FIGURE 1. Anomalous aortic origin of a coronary artery arising from the inappropriate sinus: Course subtypes. The 5 main course subtypes of anomalous aortic origin of a coronary artery arising from the inappropriate sinus are shown: blue: pre-pulmonic; orange: subpulmonic; green: retroaortic; purple: retrocardiac. the proximal segment of the interarterial course can be intramural (within the aortic wall) or not intramural. Adapted from Cheezum et al.4. Ao, aorta; MV, mitral valve; PV, pulmonic valve; TV, tricuspid valve.

and anomalous ostium at an inappropriate sinus. The five subtypes of anomalous aortic origin arising from the improper sinus can be seen in Figure $1 .^{4}$ Interarterial course is considered to be a malignant variant, with a high risk of sudden cardiac death, especially when the left coronary artery originates from the right coronary sinus. Due to its excellent temporal and spatial resolution, multidetector cardiac computed tomography angiography (CTA) allows accurate anatomical assessment of CAAs. Cardiac CTA is a Class I recommendation for initial screening of CAAs.,

\section{CASE PRESENTATION}

A 64-year-old female patient with typical chest pain was scheduled for coronary CTA due to suspected coronary artery disease. Her past medical history included obstructive sleep apnea, asthma bronchiale, and hypertension over the last 10 years. Transthoracic echocardiography (TTE) showed normal cardiac structure with preserved left and right ventricular function. The only alterations on TTE were mitral prolapse with mild mitral regurgitation, and non-significant tricuspid regurgitation without any signs of pulmonary hypertension.

Coronary CTA showed CAA: the left coronary artery originated from the right coronary sinus with a separate ostium, and it had an interarterial course with an approximately $50 \%$ stenosis on its segment between the ascending aorta and the pulmonary trunk. Moreover, the proximal segment of the left coronary artery ran within the aortic wall. Reconstruction of the coronary CTA can be seen in Figure 2. Otherwise, the patient had minimal coronary artery atherosclerosis without any other cardiac or extracardiac alterations.

The patient underwent invasive coronary angiography (ICA) for the decision-making of the surgical indication.

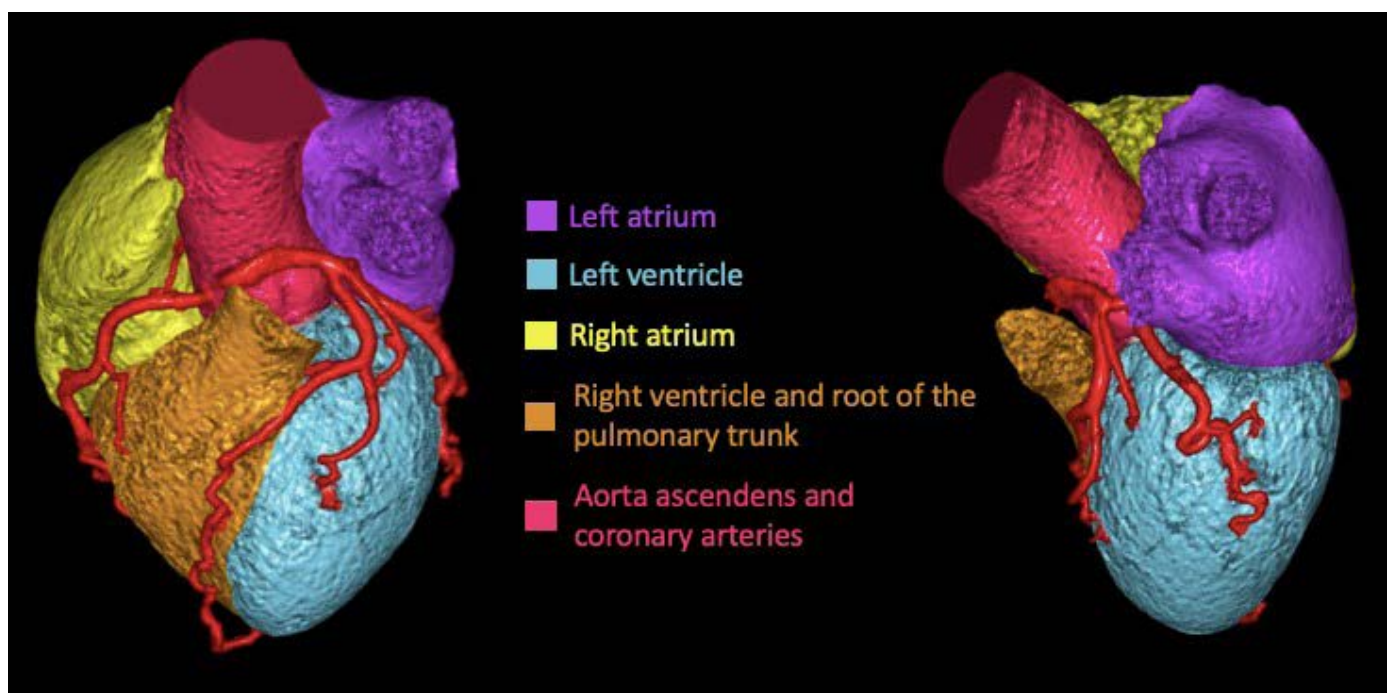

FIGURE 2. Three-dimensional volume rendered images demonstrate anomalous left coronary artery with interarterial course between ascending aorta and pulmonary trunk 
ICA confirmed the CAA with a significant stenosis on its interarterial segment. Based on the heart team's decision, the patient underwent off-pump coronary artery bypass graft (CABG) surgery. Since the proximal and middle segments of the left coronary artery ran in the myocardium, distal surgical revascularization was not possible. Therefore, two different grafts were used for the revascularization: the left internal mammary artery was connected to the first diagonal branch, and a vena saphena graft was used to connect the obtuse marginal coronary artery with the ascending aorta.

The patient agreed to the publication of her data, and the institution where the patient had been admitted approved the publication of the case.

\section{DISCUSSION}

In this report, we presented the clinical management of a patient with interarterial course left coronary artery from the first symptoms until open heart surgery. Timely diagnosis is essential for these patients, since the potential for sudden cardiac death has been largely attributed to this type of CAA. ${ }^{7}$

\section{DIAGNOSIS OF CAAS}

CAAs constitute a heterogenous group of malformations. Given the fact that $38 \%$ to $66 \%$ of CAA cases have no symptoms, timely detection of CAAs is very challenging. ${ }^{8-11}$ External compression of the abnormal course between the great arteries is especially dangerous during strenuous exercise, when the increased flow and expansion of the great vessels cause stenosis of this coronary artery segment. Therefore, the risk of sudden cardiac death is the highest among young athletes. ${ }^{8-11}$ However, due to the rarity of the cases, current guidelines do not recommend routine screening for CAAs in young patients without chest pain. ${ }^{12,13}$

The screening of young healthy individuals between 12-25 years of age with 12-lead ECG in association with comprehensive history-taking and physical examination has a Class IIb recommendation. ${ }^{13}$ Noninvasive ischemiaprovoking tests are suboptimal for the diagnosis of CAAs due to the high rate of false-negative and false-positive results. ${ }^{14,15}$

TTE is the first diagnostic option for young patients with angina. Even if TTE is a widely available, rapid noninvasive modality, it requires very experienced operators to identify coronary artery ostia, therefore TTE has limited accuracy for the detection of CAAs. In a study of 159 young patients with anomalous aortic origin of coronary arteries, there was limited correlation between TTE reports and surgical findings. ${ }^{16}$ Even if three-dimensional transesophageal echocardiography can be useful in the visualization of anomalous coronary arteries before operation, it is not a routine tool for the diagnosis of CAAs. ${ }^{17}$

Coronary CTA and magnetic resonance angiography (MRA) are the imaging modalities with a Class I recommendation for the visualization of CAAs. ${ }^{5,6}$ When comparing these two modalities, cardiac CTA is superior for imaging CAAs due to higher spatial and temporal resolution, better visualization of the surrounding structures, and lower cost. Moreover, coronary CTA we can detect coronary artery disease which might be the cause of the symptoms. Since modern CT scanners permit optimal image quality with very low radiation exposure, CTA is applicable not only for adults, but also for children with suspected CAA. ${ }^{18,19}$

Due to the limited visualization of ostium, proximal course, and surrounding structures, ICA and intravascular ultrasound (IVUS) have a Class IIa indication for the initial assessment of CAAs. ${ }^{20}$ Previous studies have shown that in $41 \%$ to $44 \%$ of the cases, ICA on its own was not sufficient for the diagnosis of CAA, and therefore patients were referred for noninvasive angiography. ${ }^{4,21}$ Fractional flow reserve (FFR) measurement along the anomalous segment can help to determine the hemodynamic significance of CAA. Prior studies have investigated the role of FFR measurement in the clinical decision-making. In a study of 37 consecutive adult patients with anomalous right coronary artery (RCA) originating from the left sinus with interarterial course, dobutamine stress FFR was significant $(\leq 0.80)$ in three patients $(8.1 \%){ }^{22}$ IVUS and optical coherence tomography can improve visualization, but further studies are needed to assess the efficacy of these techniques in this patient population.

\section{THERAPY OF CAAS}

Previous autopsy and population studies have shown that the risk of sudden cardiac death is the highest among children and adolescents during strenuous exertion, especially when the anomalous coronary artery originates from the opposite sinus with interarterial course. ${ }^{8-11,23}$ Several treatment options are available. However, there are controversial results in the literature regarding the benefit of revascularization of CAAs: while some studies suggest that surgical repair offers a safe and effective solution, , $^{3,24}$ other studies suggest that since most of these CAAs were diagnosed at an adult age without functional significance 
or cardiovascular events, consecutive management might be sufficient. ${ }^{22,25,26}$

According to the current guidelines, beyond the type of CAA, the presence of ischemic symptoms plays a central role in the decision-making: in asymptomatic patients, preventive therapy with beta-blockers and avoidance of competitive sports and strenuous physical activity are recommended. For symptomatic patients and for those with anomalous left coronary artery with an interarterial course, surgery is a Class I recommendation. ${ }^{5,27}$

Several surgical techniques are available for the treatment of CAAs including unroofing of the origin of the anomalous vessel, reimplantation to the correct sinus, and CABG operation. ${ }^{5}$ By unroofing the anomalous orifice, we can reduce the lateral compression on the intramural segment by reducing the take-off angle of the anomalous coronary artery. However, since this technique involves manipulation of the aortic root, it can cause aortic valve instability and consequently postoperative aortic insufficiency. ${ }^{24,28,29}$ Moreover, this method does not address the interarterial coronary artery segment. Reimplantation of the anomalous ostium has also been proposed, but stenosis of the new ostium can occur, resulting in reoperation and need of CABG surgery..$^{30}$ However, while some authors reported CABG to be the most straightforward solution, ${ }^{31,32}$ other studies suggest that CABG should be avoided in the absence of concomitant obstructive coronary artery disease, because competitive flow from native vessels can cause graft failure. ${ }^{4}$

There is only limited evidence on the role of percutaneous coronary intervention (PCI) in patients with interarterial coronary artery segment. Even if stent implantation can relieve compression of the interarterial course, performance of PCI can be challenging in these patients. Moreover, in a study of 42 adult patients with this type of CAA, $29 \%$ had recurrent symptoms, while the rate of in-stent restenosis was $13 \%$ after a median follow-up of 5 years. ${ }^{33} \mathrm{PCI}$ and stent implantation is currently not recommended by current guidelines for revascularization of the interarterial segment. ${ }^{12}$

\section{CONCLUSION}

Left coronary artery originating from the right coronary sinus with an interarterial course has a potential for sudden cardiac death. In these patients, surgical revascularization is recommended. However, timely diagnosis, especially in those without any symptoms remains a challenging issue. Among the invasive and noninvasive tests, coronary CTA proved to be the most precise modality for the detection of CAAs.

\section{CONFLICT OF INTEREST}

Nothing to declare.

\section{REFERENCES}

1. Angelini P. Coronary artery anomalies - current clinical issues: definitions, classification, incidence, clinical relevance, and treatment guidelines. Tex Heart Inst J. 2002;29:271-278.

2. Angelini P. Coronary artery anomalies: Why should we diagnose them in young athletes, by what means, and for what aims? Eur J Prev Cardiol. 2019;26:985-987. doi: 10.1177/2047487319840894

3. Angelini P. Coronary artery anomalies: an entity in search of an identity. Circulation. 2007;115:1296-1305. DOI: 10.1161/ CIRCULATIONAHA.106.618082

4. Cheezum MK, Liberthson RR, Shah NR, et al. Anomalous Aortic Origin of a Coronary Artery From the Inappropriate Sinus of Valsalva. J Am Coll Cardiol. 2017;69:1592-1608. doi: 10.1016/j.jacc.2017.01.031.

5. Warnes CA, Williams RG, Bashore TM, et al. ACC/AHA 2008 Guidelines for the Management of Adults with Congenital Heart Disease: Executive Summary: a report of the American College of Cardiology/American Heart Association Task Force on Practice Guidelines (writing committee to develop guidelines for the management of adults with congenital heart disease). Circulation. 2008;118:2395-2451. doi: 10.1161/ CIRCULATIONAHA.108.190811.

6. Taylor AJ, Cerqueira M, Hodgson JM, et al. ACCF/SCCT/ACR/ AHA/ASE/ASNC/NASCI/SCAI/SCMR 2010 Appropriate Use Criteria for Cardiac Computed Tomography. A Report of the American College of Cardiology Foundation Appropriate Use Criteria Task Force, the Society of Cardiovascular Computed Tomography, the American College of Radiology, the American Heart Association, the American Society of Echocardiography, the American Society of Nuclear Cardiology, the North American Society for Cardiovascular Imaging, the Society for Cardiovascular Angiography and Interventions, and the Society for Cardiovascular Magnetic Resonance. J Cardiovasc Comput Tomogr. 2010;4:407.e401-433. doi: 10.1016/j. jcct.2010.11.001.

7. Mirchandani S, Phoon CK. Management of anomalous coronary arteries from the contralateral sinus. Int J Cardiol. 2005;102:383-389. DOI: 10.1016/j.ijcard.2004.10.010

8. Redelmeier DA, Greenwald JA. Competing risks of mortality with marathons: retrospective analysis. BMJ. 2007;335:12751277. DOI: 10.1136/bmj.39384.551539.25.

9. Chugh SS, Reinier K, Balaji S, et al. Population-based analysis of sudden death in children: The Oregon Sudden Unexpected Death Study. Heart Rhythm. 2009;6:1618-1622. DOI: 10.1016/j. hrthm.2009.07.046.

10. Wren C, O'Sullivan JJ, Wright C. Sudden death in children and adolescents. Heart. 2000;83:410-413. DOI: 10.1136/ heart.83.4.410

11. Corrado D, Basso C, Pavei A, Michieli P, Schiavon M, Thiene G. Trends in sudden cardiovascular death in young competitive athletes after implementation of a preparticipation screening program. JAMA. 2006;296:1593-1601. DOI: 10.1001/ jama.296.13.1593 
12. Maron BJ, Levine BD, Washington RL, et al. Eligibility and Disqualification Recommendations for Competitive Athletes With Cardiovascular Abnormalities: Task Force 2: Preparticipation Screening for Cardiovascular Disease in Competitive Athletes: A Scientific Statement From the American Heart Association and American College of Cardiology. Circulation. 2015;132:e267-272. doi: 10.1161/ CIR.0000000000000238.

13. Maron BJ, Friedman RA, Kligfield P, et al. Assessment of the 12-lead electrocardiogram as a screening test for detection of cardiovascular disease in healthy general populations of young people (12-25 years of age): a scientific statement from the American Heart Association and the American College of Cardiology. J Am Coll Cardiol. 2014;64:1479-1514. doi: 10.1016/j.jacc.2014.05.006.

14. Løgstrup BB, Buhl J, Nielsen AD, Smerup MH, Nørgaard BL, Kristensen LD. Which exercise test to use for chest pain from an anomalous coronary artery. Congenit Heart Dis. 2014;9:E6E10. doi: 10.1111/chd.12046.

15. Brothers J, Carter C, McBride M, Spray T, Paridon S. Anomalous left coronary artery origin from the opposite sinus of Valsalva: evidence of intermittent ischemia. J Thorac Cardiovasc Surg. 2010;140:e27-e29. DOI: 10.1016/j.jtcvs.2009.06.029

16. Lorber R, Srivastava S, Wilder TJ, et al. Anomalous Aortic Origin of Coronary Arteries in the Young: Echocardiographic Evaluation With Surgical Correlation. JACC Cardiovasc Imaging. 2015;8:1239-1249. doi: 10.1016/j.jcmg.2015.04.027.

17. Yilmaz H, Gungor B, Sahin S, Bolca O. A Case of Anomalous Origin of Circumflex Artery from Right Sinus of Valsalva Recognized by Three-dimensional Transesophageal Echocardiography and Coronary Computed Tomography Angiography. Heart Views. 2014;15:57-59. doi: 10.4103/1995705X.137510.

18. Ghoshhajra BB, Lee AM, Engel LC, et al. Radiation dose reduction in pediatric cardiac computed tomography: experience from a tertiary medical center. Pediatr Cardiol. 2014;35:171-179. doi: 10.1007/s00246-013-0758-5.

19. Doss M. Pediatric Computed Tomography Scans and Cancer Risk. JAMA Pediatr. 2018;172:1099-1100. doi: 10.1001/ jamapediatrics.2018.2657.

20. Feltes TF, Bacha E, Beekman RH, et al. Indications for cardiac catheterization and intervention in pediatric cardiac disease: a scientific statement from the American Heart Association. Circulation. 2011;123:2607-2652. doi: 10.1161/ CIR.ob013e31821b1f10.

21. Barriales-Villa R, Morís C, Sanmartín JC, Fernández E, Pajín F, Ruiz Nodar JM. [Anomalous coronary arteries originating in the contralateral sinus of Valsalva: registry of thirteen Spanish hospitals (RACES)]. Rev Esp Cardiol. 2006;59:620-623.
22. Lee SE, Yu CW, Park K, et al. Physiological and clinical relevance of anomalous right coronary artery originating from left sinus of Valsalva in adults. Heart. 2016;102:114-119. doi: 10.1136/heartjnl-2015-308488.

23. Frescura C, Basso C, Thiene G, et al. Anomalous origin of coronary arteries and risk of sudden death: a study based on an autopsy population of congenital heart disease. Hum Pathol. 1998;29:689-695. DOI: 10.1016/s0046-8177(98)90277-5.

24. Mainwaring RD, Reddy VM, Reinhartz O, Petrossian E, Punn R, Hanley FL. Surgical repair of anomalous aortic origin of a coronary artery. Eur J Cardiothorac Surg. 2014;46:20-26. doi: 10.1093/ejcts/ezt614.

25. Lytrivi ID, Wong AH, Ko HH, et al. Echocardiographic diagnosis of clinically silent congenital coronary artery anomalies. Int J Cardiol. 2008;126:386-393. DOI: 10.1016/j.ijcard.2007.04.063

26. Krasuski RA, Magyar D, Hart S, et al. Long-term outcome and impact of surgery on adults with coronary arteries originating from the opposite coronary cusp. Circulation. 2011;123:154162. doi: 10.1161/CIRCULATIONAHA.109.921106.

27. Hillis LD, Smith PK, Anderson JL, et al. 2011 ACCF/AHA Guideline for Coronary Artery Bypass Graft Surgery: a report of the American College of Cardiology Foundation/American Heart Association Task Force on Practice Guidelines. Circulation. 2011;124:e652-735. doi: 10.1161/CIR.ob013e31823b5fee.

28. Furukawa K, Itoh T. Direct coronary reimplantation for repair of anomalous aortic origin of left or right coronary artery. Ann Thorac Surg. 2005;79:389-390. DOI: 10.1016/j. athoracsur.2003.12.087

29. Romp RL, Herlong JR, Landolfo CK, et al. Outcome of unroofing procedure for repair of anomalous aortic origin of left or right coronary artery. Ann Thorac Surg. 2003;76:589-595. DOI: 10.1016/s0003-4975(03)00436-3

30. van Son JA, Mohr FW. Modified unroofing procedure in anomalous aortic origin of left or right coronary artery. Ann Thorac Surg. 1997;64:568-569. DOI: 10.1016/S00034975(97)00447-5

31. Ibraheem WI, Abass OA, Toema AM, Yehia AM. Coronary artery bypass grafting experience in the setting of an anomalous origin of the right coronary artery from the left sinus of Valsalva: Midterm results. J Card Surg. 2019;34:11621171. https://doi.org/10.1111/jocs.14234

32. Sarfaraz ZK, Siddiqi MS, Al-Kindi AH, Alameddine T. Anomalous Origin of the Right Coronary Artery from the Left Coronary Sinus: Case report. Sultan Qaboos Univ Med J. 2017;17:e352-e354. doi: 10.18295/squmj.2017.17.03.017.

33. Angelini P, Uribe C, Monge J, Tobis JM, Elayda MA, Willerson JT. Origin of the right coronary artery from the opposite sinus of Valsalva in adults: characterization by intravascular ultrasonography at baseline and after stent angioplasty. Catheter Cardiovasc Interv. 2015;86:199-208. doi: 10.1002/ccd.26069. 